\title{
PERFORMANCE COMPARISON OF SINGLE AND MULTI-CHANNEL CSMA-CD WIRELESS NETWORKS USING EQUILIBRIUM POINT ANALYSIS
}

\author{
F. L. Lo \\ fllo@hkueee.hku.hk \\ T. S. Ng \\ tsng@hkueee.hku.hk \\ T. I. Yuk \\ tiyuk@hkueee.hku.hk \\ Department of Electrical and Electronic Engineering \\ The University of Hong Kong, Pokfulam Road, Hong Kong \\ Tel.: (852) 2857-8406 Fax.: (852) 2559-8738
}

\begin{abstract}
A fundamental defect of simple CSMA-CD multiple access scheme is that messages meant for different stations can collide in the common channel. In this paper, a multi-channel form of CSMA-CD network, created by CDMA signalling, is analyzed. Its performance is shown to be superior to that of a single channel CSMA-CD network.
\end{abstract}

\section{INTRODUCTION}

In the early days of radio communication between computers, networks were simple, consisting of a number of terminals sending occasional queries to a central computer, and ALOHA channel access [1] was efficient enough to handle the data traffic. As computer networks become more complex, and applications and databases are spreaded over several stations, data traffic between the different stations increases, making simple slotted ALOHA with its maximum throughput of $1 / e$ unable to provide sufficient link capacity. More efficient multiple access protocols such as CSMA (Carrier-sense Multiple Access) [2] and CSMA-CD (CSMA with Collision Detection) [3] are used to provide better throughput-delay performance for these networks. An inherent deficiency of these two multiple-access protocols is the possibility of collisions of packets meant for different stations since only one channel of transmission exists.

Additional channels can be created using FDMA or TDMA, and such systems are shown to be able to provide better performance [4], but this improvement in performance is paid for dearly in terms of the much more complex equipment required, and also the higher bandwidth which need to be set aside even when not used. A much better way of obtaining several channels for data transmission, while not sacrificing the simplicity of ALOHA access is through the use of spread-spectrum signalling. Each station can be given a separate spreading code, as in [5], or a different phase of the same spreading sequence if the system is synchronized [6], with which to receive messages. If the spread-spectrum channel is lightly loaded, the signals for the different stations will not interfere greatly with one another because of the low cross-correlation between the spreading codes, and therefore each station will seem to have a separate private 'virtual' channel. Unlike channels created by FDMA and TDMA, these 'virtual' channels created with CDMA (Code Division Multiple Access) cost little when not used. Signals in a lightly loaded spreadspectrum channel look just like low-level wide-band noise to other narrow-band radio equipment. This is the reason for the popularity of low-power spread-spectrum transceivers working in the ISM band, e.g. [7].
In this paper, single-channel as well as multi-channel CSMA$\mathrm{CD}$ networks with slotted time and variable length messages will be studied using equilibrium point analysis (EPA). EPA was first used to analyze the behavior of ALOHA networks in [8], where it was called fluid-flow approximation. The idea behind EPA is that in the long run a system will stay near the equilibrium point in the system state space where message input balances message output. This point provides the equilibrium throughput and number of blocked stations for the network, which are then taken as the average values for the two quantities. EPA has been shown to work well when there is only one intersection point between the input message curve, also called the load line, and the output message curve. When there are more than one intersection point, the system is said to be unstable because it will wander from one equilibrium point to another with the passage of time. EPA thus allows us to determine whether a system is stable, and if it is so, what the average throughput and delay of the system are.

This paper is organized as follows. Section II will present the EPA of a single channel CSMA-CD network while Section III will contain the results of the analysis. Section IV will present EPA of multi-channel CSMA-CD networks, while the results will appear in Section V. The paper ends with Section VI where comparisons between the two networks are made, and some conclusions are provided

\section{EPA OF SINGLE CHANNEL NETWORKS}

Let us consider a single channel CSMA-CD network with $N$ stations. Time is divided into mini-slots of duration $\tau$, each of sufficient length for signal transmitted by any station to be detected by all others, and also for all affected transmitting stations to abort transmission should a collision between messages occur. When a station is waiting for new messages to be generated, it is called idle. Idle stations are presented with newly generated messages at the rate of $s$ messages per mini-slot. Once a station is presented with a new message, it will listen to the common channel, and if it detects that the channel is unused, will transmit in the next mini-slot with probability one. If no other stations transmit at the same mini-slot, then the station is said to have captured the channel, and will continue to successfully transmit the message. Messages consist of a random number of mini-packets, each taking $\tau$ to transmit, which is geometrically distributed with mean $l$.

If two or more stations transmit during the same mini-slot, the messages will collide and be destroyed. All transmitting stations will be aware of it, and cease transmission within the same mini-slot. Stations with aborted messages are said to be blocked, and will not 
accept newly-generated messages. Blocked stations will listen to the channel continuously, and if the channel is detected to be silent, will retransmit with probability $p$ in the following mini-slot. Stations with newly generated messages that detect a busy channel will also be called blocked, and will follow the same procedure of listening and retransmission.

All timing imperfections, multipath fading, noise and other sources of interference are neglected, and messages sent that do not collide are assumed to be received accurately. Assume now that the system is in equilibrium with $n_{b e}$ blocked stations. The number of idle stations is $n_{o e}=N-n_{b e}$. The rate at which new messages are generated is $S_{i n}=n_{o e} s$. When the channel is captured, no stations can capture the channel. When the channel is free, the capture rate is

$$
\begin{aligned}
S_{c a p}(\text { free })= & n_{o e} s(1-s)^{n_{o e}-1}(1-p)^{n_{b e}} \\
& +n_{b e} p(1-p)^{n_{b e}-1}(1-s)^{n_{o e}} .
\end{aligned}
$$

The channel will remain captured on average $l+1$ mini-slots, as it takes $l$ mini-slots on average for the message to be completely transmitted, and an extra one mini-slot for all the stations to realize that the channel is now free. The channel will remain free on average $l_{f}=1 / S_{\text {cap }}$ (free) mini-slots. The average capture rate of the channel is therefore

$$
S_{\text {cap }}(\text { average })=l_{f}\left(l+1+l_{f}\right)^{-1} S_{\text {cap }}(\text { free })=\left(l+1+l_{f}\right)^{-1} .
$$

Since any station capturing the channel is guaranteed to successfully complete transmitting its message, therefore the average capture rate is also the average message output rate $S_{\text {out }}$.

Thus by plotting $S_{i n}$ and $S_{c a p}$ (average) against $n_{b}$, we can determine the equilibrium point(s) by noting the intersection(s) of the two curves. Following [8], we can have the following situations. If there is only one intersection point where $n_{b}$ is much less than $N$, then the system is stable with high throughput. If more than one intersection points exist, then the system is not stable as the system will move from one stable equilibrium point to another with the passage of time. If only one equilibrium point exists at $n_{b}$ near $N$, then the system is said to be congested, with very low throughput and very high delay. The equilibrium delay, $d$, can be obtained from $S_{\text {out }}$ and $n_{b}$ by Little's Formula, $d=n_{b} / S_{\text {out }}$.

Note that equilibrium points with $S_{\text {in }}<S_{\text {out }}$ to the left and $S_{\text {in }}>S_{\text {out }}$ to the right are stable equilibrium points since any movement away from them will cause the system to shift back towards those points. The other type of equilibrium point is unstable.

\section{RESULT FOR SINGLE-CHANNEL SYSTEM}

Fig. 1 shows the $S_{\text {in }}$ and $S_{\text {out }}$ curves of a single-channel CSMA-CD system with $N=50, s=0.001$ and $l=20$. When $p=0.1$, the system is stable with high throughput. When $p=0.15$, the system is unstable, but there is a big separation between the first two equilibrium points, meaning that the system will require a long time to move from one to the other. When $p=0.2$, the separation between the first two equilibrium points is much smaller. When $p=0.22$, the system is congested and throughput drops to a very low number.

Table 1 shows the throughputs and delays of the system obtained by EPA and simulation for 100,000 mini-slots for the various values of $p$ plotted in Fig. 1. When the system is stable with $p=0.10$, the throughput and delay values obtained from EPA match those obtained from simulation, as expected. For $p=0.15$, simulation over 100,000 mini-slots is insufficient to allow the system to reach the second equilibrium point, and system performance matches that obtained from the first equilibrium point. For the $p=0.20$ case, the system enters the second stable equilibrium point easily, and system performance drops drastically. For the congested $p=0.22$ case, both EPA and simulation provide very low throughput and very high delay values.

\section{EPA OF MULTI-CHANNEL NETWORKS}

We shall again consider a network of $N$ stations, with minislotted time and messages made up of a geometrically distributed random number of packets with a mean of $l$. Messages generated at a station are equally likely to be intended for any other station. Each station is assigned a specific spreading code, or a specific phase of the same spreading code if the system is synchronized, with which it will receive messages. Thus each station is seen to possess a 'virtual' channel for reception of messages. A station with no messages to transmit is said to be idle. Idle stations are presented with newly generated messages at a rate of $s$ messages per mini-slot. A station with a newly generated message for station $k$ will first check if channel $k$ is quiet. If it is, the station will transmit during the next mini-slot with probability one. If no other stations start transmission down the same channel during that mini-slot, then the station is said to have captured the channel and can successfully complete the transmission of the whole message. Should two or more stations transmit down the same channel $k$ during the same mini-slot, then a collision of the messages is said to have occurred, and all stations involved will detect this and abort transmission of the message in the same mini-slot. Such blocked stations will refuse any newly generated messages and continue listening to channel $k$. Following each mini-slot when the channel is determined to be free, the blocked stations will attennpt transmission with probability $p$. Stations with newly generated messages that detect the channel of the intended receiver to be busy will also be blocked, and will follow the same retransmission procedure. For ease of discussion, we shall call channels which have blocked stations 'occupied'.

First, we wish to determine if a system will be stable and uncongested. Consider the situation where an occupied channel $k$ has $n_{b k}$ blocked stations, and there are $n_{o}$ idle stations in the system. When the channel $k$ is captured, on average, per mini-slot, the number of idle stations that will be presented with a newly generated message intended for station $k$ is

$$
\begin{aligned}
& \Delta n_{b k}(c a p) \\
& =n_{o} \frac{s}{N}\left(1-\frac{s}{N}\right)^{n_{o}-1}+2\left(\begin{array}{c}
n_{o} \\
2
\end{array}\right)\left(\frac{s}{N}\right)^{2}\left(1-\frac{s}{N}\right)^{n_{o}-2} \\
& +3\left(\begin{array}{c}
n_{o} \\
3
\end{array}\right)\left(\frac{s}{N}\right)^{3}\left(1-\frac{s}{N}\right)^{n_{o}-3}+\cdots+n_{o}\left(\frac{s}{N}\right)^{n_{o}} .
\end{aligned}
$$

This is also, of course, the average number of additional blocked stations that will appear at the channel $k$.

When the channel is free, on average per mini-slot, the additional number of blocked stations that will appear at the channel $k$ is 


$$
\begin{aligned}
& \Delta n_{b k} \text { (freet) } \\
& =n_{o} \frac{s}{N}\left(1-\frac{s}{N}\right)^{n_{o}-1}\left[1-(1-p)^{n_{b k}}\right]+2\left(\begin{array}{c}
n_{o} \\
2
\end{array}\right)\left(\frac{s}{N}\right)^{2}\left(1-\frac{s}{N}\right)^{n_{o}-2} \\
& +3\left(\begin{array}{c}
n_{o} \\
3
\end{array}\right)\left(\frac{s}{N}\right)^{3}\left(1-\frac{s}{N}\right)^{n_{o}-3}+\cdots n_{o}\left(\frac{s}{N}\right)^{n_{o}}
\end{aligned}
$$

When the channel is free, on average per mini-slot, the decrease in the number of blocked stations is just the probability that a blocked station will capture the channel. This is

$$
\Delta n_{b k}(\text { free- })=n_{b k} p(1-p)^{n_{b k}-1}\left(1-\frac{s}{N}\right)^{n_{o}} .
$$

On average, a channel will remain captured for $l+1$ mini-slots and it will remain free for $l_{f}$ mini-slots, with $l_{f}=1 / p_{f}$, where $p_{f}$ is the probability that a station will capture the channel,

$$
\begin{aligned}
p_{f}= & n_{o} \frac{s}{N}\left(1-\frac{s}{N}\right)^{n_{o}-1}(1-p)^{n_{b k}} \\
& +n_{b k} p(1-p)^{n_{b k}-1}\left(1-\frac{s}{N}\right)^{n_{o}} .
\end{aligned}
$$

Thus, on average, the number of additional blocked stations that will appear at the blocked channel $k$ per mini-slot is just

$$
\begin{aligned}
& \Delta n_{b k}=(l+1) \cdot\left(l+1+l_{f}\right)^{-1} \cdot\left[\Delta n_{b k}(c a p)\right] \\
& +l_{f} \cdot\left(l+1+l_{f}\right)^{-1} \cdot\left[\Delta n_{b k}(\text { free }+)-\Delta n_{b k}(\text { free }-)\right] .
\end{aligned}
$$

For the system to be at equilibrium, the average message input $S_{\text {in }}=n_{o} s$ must be equal to the average message completion $S_{\text {out }}=n_{t} /(l+1)$ where $n_{t}$ is the number of transmitting stations. Thus at equilibrium, the following equation must be satisfied

$$
\left(N-n_{b}-n_{t}\right) s=\frac{n_{t}}{l+1} \quad \text { or } \quad n_{t}=\frac{s\left(N-n_{b}\right)}{s+(l+1)^{-1}} .
$$

To determine whether a system at equilibrium is stable, we examine all the points where (8) is satisfied, and calculate $\Delta n_{b k}$ given by (7) for $n_{b k}=\left\{1,2,3, \ldots, n_{b}\right\}$, and for all $n_{b}$. If all the $\Delta n_{b k}$ 's are negative, then the system is stable since any occupied channel will tend to clear itself of blocked stations. For cases where all $\Delta n_{b k}$ 's are positive, then the system is congested, as all occupied channels tend to accumulate blocked stations, and thus lower the message input. For cases where $\Delta n_{b k}$ 's can be positive or negative, the system is unstable, since it can remain at points in phase space where message input is high or at points where most of the stations are blocked.

Once we have determined that a system is stable and uncongested, we have to estimate its throughput and delay. If a system is in equilibrium, as we have seen, the number of transmitting stations and blocked stations must be related by (8). We have to find out the point on the equilibrium curve determined by this equation where the system will settle down. To do this, we have to calculate the equilibrium capture rate. The capture rate of the system given $n_{t}$ and $n_{b}$ depends on how the blocked stations are distributed among the occupied channels. We have already seen that for the system to be uncongested and stable, the occupied channels will tend to clear themselves. Thus it is reasonable to assume that at equilibrium, stable and uncongested networks will have only one blocked station per occupied channel. The results presented in the next section shows that this assumption is valid.

Suppose there are $n_{b}$ blocked stations distributed one to each occupied channel. For occupied channels, the capture rate when the channel is free is

$$
S_{\text {cap }}(\text { occ })=n_{o} \frac{s}{N}\left(1-\frac{s}{N}\right)^{n_{o}-1}(1-p)+\left(1-\frac{s}{N}\right)^{n_{o}} p .
$$

On average the occupied channel will stay free for $l_{c}=1 / S_{c a p}(o c c)$ mini-slots. For an unoccupied channel, the capture rate when the channel is free is just

$$
S_{\text {cap }}(\text { unocc })=n_{o} \frac{s}{N}\left(1-\frac{s}{N}\right)^{n_{o}-1} .
$$

On average the unoccupied channel will stay free for $l_{u}=1 / S_{\text {cap }}($ unocc $)$ mini-slots.

When a channel is captured, its capture rate of course drops to zero. Furthermore, a channel will stay captured for $l+1$ mini-slots on average. Thus the average capture rate of a stable, uncongested system at equilibrium is

$$
\begin{aligned}
& S_{\text {cap }} \text { (average) } \\
& =n_{b} \frac{l_{c}}{l_{c}+l+1} S_{c a p}(\text { occ })+\left(N-n_{b}\right) \frac{l_{u}}{l_{u}+l+1} S_{c a p}(\text { unocc }) \\
& =n_{b} \frac{1}{l_{c}+l+1}+\left(N-n_{b}\right) \frac{1}{l_{u}+l+1}
\end{aligned} .
$$

When we plot $S_{\text {cop }}$ (average) and the $S_{\text {in }}=S_{\text {out }}$ curve against $n_{b}$ and $n_{t}$ related by (8), we will get just one intersection. For stable and uncongested system, this point gives the equilibrium throughput $S_{\text {out }}$ and number of blocked stations $n_{b}$. Using Little's Theorem, we can find the equilibrium delay, $d=n_{b} / S_{\text {out }}$.

\section{RESULTS FOR MULTI-CHANNEL SYSTEMS}

Fig. 2 shows the $S_{\text {in }}$ and the $S_{\text {cap }}$ (average) curves for $s=0.04$, $l=20$ and the various values of $p$ 's indicated. When the system is determined to be stable, the intersection point of the two curves give the equilibrium throughput and number of blocked stations for the system.

Table 2 shows the throughput and delay results obtained from EPA and simulations over 100,000 mini-slots of a multi-channel CSMA-CD system with $N=50, l=10$ and $s=0.04$. For $p=0.10$ and $p=0.15$, the system is determined to be stable as all $\Delta n_{b k}$ 's are negative. For $p=0.20$ and $p=0.25$, the system is unstable, but the system will tend towards the congested region only when $n_{b k}$ becomes large. For the $p=0.20$ case, $n_{b k}$ must cross 27 and for $p=0.25$ case, $n_{b k}$ must cross 20 before $\Delta n_{b k}$ becomes positive. The system will therefore stay in the uncongested region for a long time before drifting into the congested part of the system phase space. The throughputs and delays obtained from simulations show that 100,000 mini-slots are not sufficient for the system to become congested.

For the $p=0.60$ case, $n_{b k}$ needs only to be greater than 6 before $\Delta n_{b k}$ becomes positive. Thus it is relatively easy for the system to move to the congested part of the system phase space. 
Simulation over 100,000 slots shows indeed very low throughput and high delay

\section{COMPARISON AND CONCLUSIONS}

As can be seen from Table 1 and 2, and also Table 3 and 4, EPA gives quite accurate values for throughput and delay for both single and multi-channel CSMA-CD networks when the system is stable and not congested. When the system is lightly loaded, e.g., with $s=0.001, l=10$, and $p=0.05$ and 0.10 , both the single as well as the multi-channel CSMA-CD networks can handle almost the maximum message generation rate, $N s=0.05$. The delays suffered by the multi-channel system, however, are much lower.

The difference in performance is even more evident when the system is more heavily loaded. For $s=0.002, l=20$ and $p=0.1$, the single channel system can handle only about $33 \%$ of the maximum possible number of messages generated, with delays suffered by successful messages going beyond 1000 mini-slots. The multichannel system, on the other hand, can still handle about $96 \%$ of the maximum possible number of messages generated, with successful messages suffering minimal delays. Even when the single channel system enter the unstable and congested region with $p=0.20$, the multi-channel system can still handle above $95 \%$ of the maximum number of messages generated, with negligible message delays.

Multi-channel CDMA CSMA-CD systems do not only provide higher throughputs and lower delays than single-channel systems. Even though they require slightly more complex transmission and reception equipment, they provide, when lightly loaded, almost no interference to other existing narrow-band users.

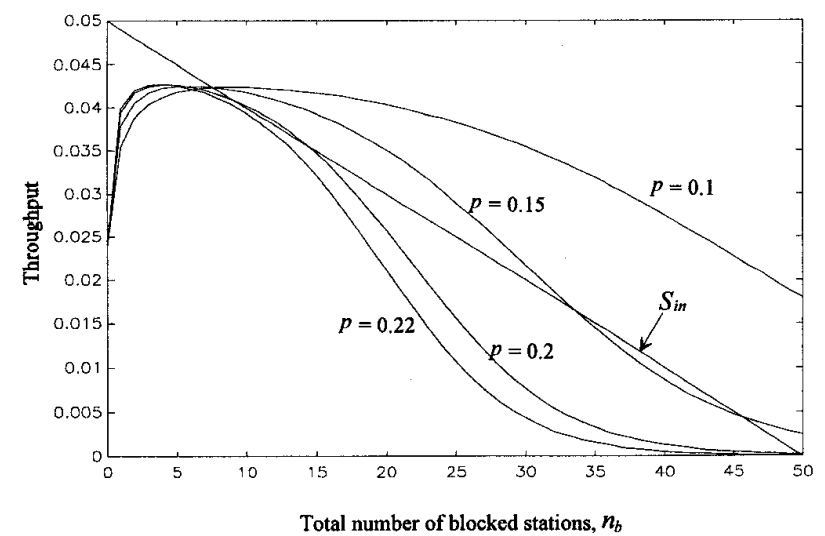

Fig. 1: Graph of the message input and average message output curves for $N=50, s=0.001, l=20$ and various values of $p$, for a single-channel system.

\section{ACKNOWLEDGMENTS}

This work was supported by HDC and the CRCG of the HKU.

\section{REFERENCES}

[1] N. Abramson, "The ALOHA System - Another Alternative for Computer Communications", Proc. AFIPS Conf., 1970 Fall Joint Comput. Conf., Vol.37, pp. 281-285, 1970.

[2] F. Tobagi, L. Kleinrock, "Packet Switching in Radio Channels: Part I Carrier Sense Multiple Access Modes and Their Throughput-Delay Characteristics", IEEE Trans. on Commun., Vol. COM-23, pp. 14001416,1975

[3] F. Tobagi, V. Hunt, "Performance Analysis of Channel Sense Multiple Access with Collision Detection", Comp. Networks, Vol. 4, pp. 245259,1980

[4] M. Marsan, D. Roffinella, "Multichannel Local Area Network Protocols", IEEE JSAC, Vol. SAC-1, pp. 885-897, 1983

[5] E. Sousa, J. Silvester, "Spreading Code Protocols for Distributed Spread-Spectrum Packet Radio Networks", IEEE Trans. on Commun., Vol. COM-36, pp. 272-281, 1988.

[6] K. Yip, T. Ng, "Code Phase Assignment - A Technique for High Capacity Indoor Mobile DS-CDMA Communication", IEEE 44th Veh. Tech. Conf., pp. 1586-1590, 1994

[7] B. Tuch, "Development of WavelAN, an ISM Band Wireless LAN", AT\&T Technical Journal, July/August 1993, pp. 27-37

[8] L. Kleinrock, S. Lam, "Packet Switching in a Multiaccess Broadcast Channel: Performance Evaluation", IEEE Trans. on Commun., Vol. COM-23, pp. 410-423, 1975.

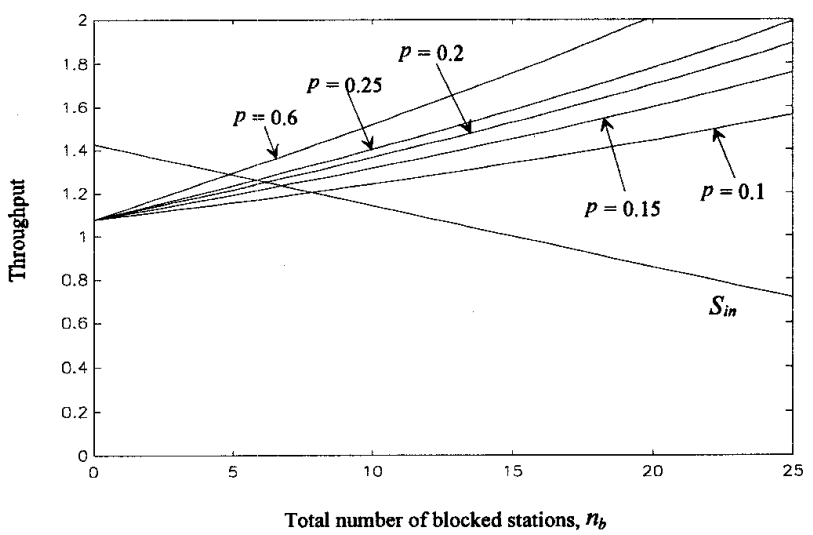

Fig. 2: Graph of the message input and the average message output curves for $N=50, s=0.04$ and $l=10$, for various values of $p$ for a multi-channel system. 


\begin{tabular}{cccccccc}
\hline & & & \multicolumn{2}{c}{ EPA } & \multicolumn{2}{c}{ Simulation } \\
$S$ & $p$ & $\ell$ & $S_{\text {cap }}$ & delay & status & thruput \\
\hline 0.001 & 0.10 & 20 & 0.0423 & 181.1 & stable & 0.0429 & 135.5 \\
0.001 & 0.15 & 20 & 0.0424 & 178.3 & unstable & 0.0421 & 156.1 \\
0.001 & 0.20 & 20 & 0.0410 & 218.3 & unstable & 0.0106 & 3639.0 \\
0.001 & 0.22 & 20 & 0.0001 & 498600 & congested & 0.0019 & 25502 \\
\hline
\end{tabular}

Table 1: Throughputs and delays for a single-channel system with $N=50, s=0.001, l=20$ and various values of $p$. Note that simulations are done over 100,000 mini-slots, and for unstable systems, the equilibrium values for the first equilibrium point are tabulated.

\begin{tabular}{cccccccc}
\hline & $p$ & & & EPA & \multicolumn{2}{c}{ Simulation } \\
$S$ & $p$ & $\ell$ & $S_{\text {cap }}$ & delay & status & thruput & delay \\
\hline 0.04 & 0.10 & 10 & 1.19 & 6.78 & stable & 1.1783 & 6.81 \\
0.04 & 0.15 & 10 & 1.23 & 5.71 & stable & 1.2009 & 6.07 \\
0.04 & 0.20 & 10 & 1.24 & 5.15 & unstable & 1.2170 & 5.52 \\
0.04 & 0.25 & 10 & 1.25 & 4.80 & unstable & 1.2295 & 5.25 \\
0.04 & 0.60 & 10 & 1.28 & 3.97 & unstable & 0.0500 & 965.02 \\
\hline
\end{tabular}

Table 2: Throughputs and delays for a multi-channel system with $N=50, s=0.04, l=10$ and various values of $p$. Simulations are done over 100,000 mini-slots.

\begin{tabular}{cccccccc}
\hline$S$ & $p$ & $\ell$ & $S_{\text {cap }}$ & EPA & \multicolumn{2}{c}{ Simulation } \\
delay & status & delay \\
\hline 0.001 & 0.05 & 10 & 0.0487 & 26.9 & stable & 0.0495 & 17.4 \\
0.001 & 0.05 & 20 & 0.0412 & 213.6 & stable & 0.0418 & 156.7 \\
0.001 & 0.10 & 10 & 0.0494 & 13.2 & stable & 0.0496 & 15.8 \\
0.001 & 0.10 & 20 & 0.0423 & 181.1 & stable & 0.0423 & 148.0 \\
0.002 & 0.05 & 10 & 0.0728 & 186.5 & stable & 0.0718 & 159.1 \\
0.002 & 0.05 & 20 & 0.0417 & 697.8 & stable & 0.0420 & 626.0 \\
0.002 & 0.10 & 10 & 0.0720 & 194.2 & stable & 0.0716 & 167.3 \\
0.002 & 0.10 & 20 & 0.0329 & 1019.2 & stable & 0.0335 & 933.6 \\
0.001 & 0.20 & 20 & 0.0411 & 215.3 & unstable & 0.0057 & 7712.5 \\
0.002 & 0.20 & 20 & 0.0002 & 249400 & congested & 0.0008 \\
\hline
\end{tabular}

Table 3: Throughputs and delays for a single-channel system with $N=50$, and various values of $S, p$ and $l$. Note that simulations are done over 100,000 mini-slots, and for the unstable system, the equilibrium values for the first equilibrium point are used.

\begin{tabular}{cccccccc}
\hline & & & & EPA & \multicolumn{2}{c}{ Simulation } \\
$S$ & $p$ & $\ell$ & & delay & status & thruput & delay \\
\hline 0.001 & 0.05 & 10 & 0.0495 & 0.40 & stable & 0.050 & 0.01 \\
0.001 & 0.05 & 20 & 0.0490 & 1.02 & stable & 0.050 & 0.15 \\
0.001 & 0.10 & 10 & 0.0495 & 0.20 & stable & 0.050 & 0.00 \\
0.001 & 0.10 & 20 & 0.0490 & 0.61 & stable & 0.050 & 0.15 \\
0.002 & 0.05 & 10 & 0.0979 & 0.71 & stable & 0.100 & 0.01 \\
0.002 & 0.05 & 20 & 0.0958 & 1.77 & stable & 0.100 & 0.01 \\
0.002 & 0.10 & 10 & 0.0979 & 0.51 & stable & 0.100 & 0.01 \\
0.002 & 0.10 & 20 & 0.0959 & 1.35 & stable & 0.098 & 0.01 \\
0.001 & 0.20 & 20 & 0.0490 & 0.61 & stable & 0.050 & 0.00 \\
0.002 & 0.20 & 20 & 0.0959 & 1.15 & stable & 0.098 & 0.01 \\
\hline
\end{tabular}

Table 4: Throughputs and delays for a multi-channel system with $N=50$, and various values of $S, p$ and $l$. Note that simulations are done over 100,000 mini-slots. 